\title{
Concurrent chemobiotherapy with cisplatin, dacarbazine, decrescendo interleukin-2 and interferon $\alpha 2 b$ in patients with metastatic melanoma
}

\author{
JAIR BAR ${ }^{1,4^{*}}$, RINAT YERUSHALMI ${ }^{2,4 *}$, RONI SHAPIRA-FRUMMER ${ }^{1 *}$, IRENA KUTCHUK $^{1}$, \\ AARON SULKES ${ }^{2,4}$, HAIM GUTMAN ${ }^{3,4}$, RAPHAEL CATANE $^{1,4}$ and JACOB SCHACHTER ${ }^{1,4}$ \\ ${ }^{1}$ Division of Oncology, Chaim Sheba Medical Center (CSMC), Tel-Hashomer 52621; ${ }^{2}$ Institute of Oncology, \\ Davidoff Center, Rabin Medical Center (RMC), ${ }^{3}$ Department of Surgery B, RMC, Jabotinsky 39, Petah Tikva 49100; \\ ${ }^{4}$ Sackler Faculty of Medicine, Tel-Aviv University, Ramat Aviv, Tel-Aviv 69978, Israel
}

Received May 20, 2008; Accepted July 19, 2008

DOI: 10.3892/or_00000176

\begin{abstract}
We aimed to evaluate a concurrent chemobiotherapy (CBT) regimen consisting of cisplatin (CDDP), dacarbazine (DTIC), decrescendo interleukin-2 (IL-2), and interferon $\alpha 2 b$ (INF- $\alpha 2 b$ ), in metastatic melanoma patients. A total of 60 patients with biopsy proven, metastatic melanoma were treated between October 2000 and November 2005 at the Oncology Institutes of RMC and CSMC. Patients received concurrent CBT for 5 days, consisting of CDDP, DTIC, decrescendo IL-2, and subcutaneous INF- $\alpha 2 b$. GM-CSF was given subcutaneously on days 8 to 12 of each cycle, to the first 26 patients. Treatment was administered q21d for a total of six cycles or until severe toxicity or progression; 57 patients who received at least two cycles, followed for at least 24 months, were included in response analysis. The overall response rate (RR) reached $44 \%$ (28/57 patients); 14 patients had a complete response $(\mathrm{CR}, 25 \%) ; 11(19 \%)$ reached a partial response. The median progression-free survival was 7 months. Median overall survival (OS) was 11.7 months. At a median followup of 29 months, 8 of 14 complete responders remain alive for more than two years, with no clinical evidence of disease. Median OS of patients with CR has not been reached; $17 \%$ of the courses were modified due to toxicity, and $20 \%$ of the patients were removed from the protocol due to toxicity or refusal to continue. The data from this study indicate that this protocol of concomitant CBT is feasible with a fraction of the patients achieving a durable CR.
\end{abstract}

Correspondence to: Dr Jacob Schachter, Oncology Institute, CSMC, 52621 Tel-Hashomer, Israel

E-mail: jacob.schachter@sheba.health.gov.il

${ }^{*}$ Contributed equally

Key words: metastatic melanoma, chemobiotherapy, complete response

\section{Introduction}

Chemotherapy with a single agent such as DTIC can induce objective tumor regression in only $10-20 \%$ of patients with metastatic melanoma, without a meaningful survival advantage. Combination chemotherapy can achieve a response rate (RR) of about $30 \%$ but again has not shown a survival advantage over single agent chemotherapy (1). Biological response modifiers have been implemented as an alternative strategy for metastatic melanoma patients. IL-2 and INF- $\alpha$ have yielded an RR in the range of $15-20 \%$. However, in a small cohort of patients durable complete remissions have been reported, mainly in patients receiving bolus high dose IL-2 (2). High-dose IL-2 is a highly toxic regimen, leading to up to a $2 \%$ death rate from adverse events (3). On the other hand, a decrescendo dose-schedule of IL-2 has been shown to reduce toxicity but not efficacy (4). INF- $\alpha$ is also associated with multiorgan toxicity such as 'flu-like' syndrome and autoimmune manifestations, but its toxicity is almost never associated with the need for hospitalization.

Combining chemotherapy with immunotherapy may be advantageous. Chemotherapy-induced apoptosis of cancer cells activates in some cases an immunologic response that contributes to regression of established tumors (5). The mechanisms proposed for the induction of an anti-tumor immune response by chemotherapy include augmented release of tumor antigens, damaged DNA as a strong immunogen, and induction of death receptors (Fas and TRAIL) by some chemotherapy agents (6). On the other hand, this immunologic response may be inhibited by the use of high-dose chemotherapy or by certain chemotherapeutic drugs. Augmenting the immune response by the concurrent use of immune modulators such as IL-2 and INF may be a rational approach for maximizing the efficacy of treatments (7).

In accordance to this rational, in an attempt to maximize response, and due to the apparent lack of cross-resistance, the combination of chemotherapy and biotherapy was assessed in a few phase II clinical trials in metastatic melanoma patients. 
The RR in those studies was in the range of $50 \%$. Most importantly, a durable complete response was achieved in $\sim 10 \%$ of the patients. The toxicity of combined chemotherapy and biotherapy (CBT) protocols was milder than treatment with high dose IL-2 (8). One phase III trial suggested significant benefit for CBT compared to chemotherapy alone (9). However, other phase III trials did not demonstrate a survival advantage for CBT compared to chemotherapy alone (10-13). A large meta-analysis was recently reported (14), which concluded that although CBT increases RR, this does not translate into a survival benefit. All reported trials examined a cisplatin-based chemotherapy regimen, with or without interferon, or with or without interferon and IL-2. It should be noted that many of the studies that evaluated CBT did not administer all components concomitantly (9-12). In addition, the doses of IL-2 administered were relatively low in many of these studies (12), either according to the treatment plan, or secondary to dose reductions following toxicity of the treatment. These low doses of IL-2 in CBT treatments do not have the anti-melanoma efficacy of high-dose IL-2 (15). The administration of concomitant CBT requires dedicated oncology teams. Large multi-center trials probably include Medical Centers that are not intimately familiar with this treatment, and thus may not truly reflect the efficacy of this treatment.

In order to explore the benefits of CBT, we conducted a retrospective analysis of our data. We report our experience with a 5-day concurrent CBT consisting of CDDP, DTIC, IL-2, INF- $\alpha 2 b$, and in some of the patients, GM-CSF as an immunomodulator, administered at three-week intervals. We have used a decrescendo IL-2 schedule based on studies suggesting that this dosing improves efficacy and reduces cumulative toxicity (16). GM-CSF-treated patients received a 7-day course of granulocyte macrophage colony stimulating factor on the second week of the cycle as an immunomodulator $(17,18)$.

\section{Patients and methods}

Between October 2000 and November 2005, 60 patients received $\mathrm{CBT}$ as first line treatment for metastatic melanoma; 26 consecutive patients received the CBT treatment at the Oncology Institute of RMC, and an additional 34 consecutive patients were treated at the Oncology Institute of CSMC. All patients were treated under the supervision of the same P.I. (J.S.). All available medical records and researcher files were retrospectively reviewed. Data of five patients were not fully retrievable and thus have been included only in the RR, PFS and OS analysis. In these five patients no CR was observed, and OS was 3-12 months; 57 patients that received at least two cycles, with a minimum follow-up of 24 months (from the onset of the first cycle of CBT) were included in the response analysis. CBT was given to patients with biopsy proven, inoperable metastatic melanoma, aged 18-75 years, with measurable disease. Prior radiotherapy was given to some patients and was completed at least 3 weeks before starting CBT.

Additional requirements were: performance status 0-2 (ECOG), white blood cell count $>3.000 / \mathrm{mm}^{3}$, platelet count
Table I. Treatment regimen.

\begin{tabular}{|c|c|c|c|c|c|}
\hline \multicolumn{6}{|l|}{ 1st week } \\
\hline Rx/day & 1 & 2 & 3 & 4 & 5 \\
\hline IV Cisplatin (mg/m²) & 25 & 25 & 25 & - & - \\
\hline IV Dacarbazin $\left(\mathrm{mg} / \mathrm{m}^{2}\right)$ & 220 & 220 & 220 & - & - \\
\hline IV IL-2 (MIU/m²) & 18 & 9 & 4.5 & 4.5 & - \\
\hline SC INF $\alpha 2 b(\mathrm{MIU})$ & 5 & 5 & 5 & 5 & 5 \\
\hline
\end{tabular}

2nd week (for the RMC cohort):

S.C. GM-CSF $150 \mu \mathrm{g} /$ day-days 8-12

3rd week

Rest

$>100,000 / \mathrm{mm}^{3}$, normal creatinine level, bilirubin level $<2.0 \mathrm{mg} / \mathrm{dl}$ and normal thyroid function tests. Patients $>50$ years old or any symptomatic patients had to have a normal cardiac evaluation (normal ECG and Thalium 201 effort stressed cardiac perfusion examination). Patients with smoking history or symptomatic pulmonary dysfunction had to have pulmonary function tests with values at least $75 \%$ of predicted. Patients underwent brain MRI scan as well, and a bone scan was done if clinically indicated.

CBT was not offered to patients who were not able to stop steroid treatment, to patients with a history of a second malignancy besides non-melanoma skin cancer, and to patients with brain metastases.

All patients gave a written informed consent for the CBT treatment. This retrospective analysis was approved by the ethics committee of CSMC.

Evaluation of response and data analysis. Baseline total body CT or CT-PET was done prior to the onset of treatment. Evaluation of measurable disease was performed at the end of every two cycles as well as upon termination of treatment. Response was assessed only in patients who had completed at least 2 cycles of treatment. Complete response (CR) was defined as the disappearance of all clinical evidence of tumor by physical and radiological assessments for at least 4 weeks, during which no new lesions appeared. Partial response (PR), stable disease (SD) and progressive disease (PD) were defined according to standard criteria. Statistical analysis was performed by GraphPad Prism v.4 software. All statistical comparisons were done using logrank test and reported as two-tailed P-values.

Treatment regimen. The treatment regimen (Table I) included Cisplatin (CDDP) at a dose of $25 \mathrm{mg} / \mathrm{m}^{2} /$ day given daily for the first three days of the course. It was preceded by hydration with $1000 \mathrm{cc}$ of $\mathrm{NaCl} 0.9 \%$ for $2 \mathrm{~h}$. Dacarbazine (DTIC) was also given during the first three days of the cycle at a dose of $220 \mathrm{mg} / \mathrm{m}^{2} /$ day. Subcutaneous INF- $\alpha 2 \mathrm{~b}$ (Intron A, ScheringPlough) was given daily for 5 days at a flat dose of $5 \mathrm{mu}$ a day. IL-2 (Proleukin ${ }^{\circledR}$, Chiron) was given in a decrescendo manner to a total of $36 \mathrm{mu} / \mathrm{m}^{2}$ during the first 4 days of the 
Table II. Patient characteristics.

\begin{tabular}{lc}
\hline Median Age (years) & 54 (range 27-71) \\
Male/Female (\%) & $53 / 47$ \\
Median performance status (ECOG) & 0 (range 0-2) \\
Prior non-surgical therapy & \\
None & $30(55 \%)$ \\
Radiation therapy & $10(18 \%)$ \\
Adjuvant interferon & $14(25 \%)$ \\
Vaccine trials & $9(16 \%)$ \\
Chemobiotherapy & $1(1.8 \%)$ \\
Distribution of metastatic sites & \\
Only L.N. or soft tissue & $15(27 \%)$ \\
Lung & $26(47 \%)$ \\
Other sites & $37(67 \%)$ \\
Number of metastatic sites & \\
1 & $25(45 \%)$ \\
2 & $20(36 \%)$ \\
4 & $5(9 \%)$ \\
5 & $3(5.5 \%)$ \\
6 & $1(1.8 \%)$ \\
\hline
\end{tabular}

protocol (see Table I). In the 2000-2001 cohort (21 patients), GM-CSF (Leukomax) was given subcutaneously at a daily dose of $150 \mu \mathrm{g} /$ day on days 8-12 of the cycle. Cycles were repeated every 21 days.

Treatment cycles were delayed by 1 week if on day one the platelet count was $<100,000 / \mathrm{mm}^{3}$, or if the PMN count was $<1,000 / \mathrm{mm}^{3}$. Doses of chemotherapy were modified by $25 \%$ for dacarabazin and CDDP on subsequent cycles if the nadir platelet count was $<75,000 / \mathrm{mm}^{3}$ or absolute neutrophil count reached a nadir of $500 / \mathrm{mm}^{3}$ or less.

CBT was terminated for patients with evidence of progressive disease. Patients with stable disease received a maximum of 6 courses. Patients achieving a CR received two additional cycles of therapy, following documentation of CR, when deemed appropriate by the treating physician. Surgical resection of residual disease was performed in two patients who had a low volume of resectable residual disease. These patients were considered in our analysis as complete responders.

Supportive measures during hospitalization. Vital signs were monitored every $8 \mathrm{~h}$. Weight as well as intake/output was measured daily. During therapy, a CBC and biochemistry were monitored every day or more if indicated. Patients received hydration with $\mathrm{KCL}$ and $\mathrm{MgSO}_{4}$ supplementation at an initial rate of $100 \mathrm{cc} / \mathrm{h}$. Bolus $\mathrm{NaCl}$ $0.9 \%, 500 \mathrm{c}$, was administered when systolic blood pressure dropped to $<90 \mathrm{~mm} \mathrm{Hg}$, or when urinary output decreased below $100 \mathrm{ml} / \mathrm{h}$. Furosemide (20 mg i.v.) was administered to a maximum of two doses, followed by low dose dopamine
Table III. Toxicity of biochemotherapy.

No. of patients

\begin{tabular}{lc}
\hline Body weight gain of $>10 \%$ & $8(13)$ \\
Systolic hypotension $(<90 \mathrm{~mm} \mathrm{Hg})$ & $8(13)$ \\
Pulmonary congestion $(\mathrm{SO} 2<90 \%)$ & $4(7)$ \\
Infection & $1(1.8)$ \\
Neutropenic fever & \\
Gastrointestinal toxicity & $4(7)$ \\
Diarrhea G 3-4 & $2(3.6)$ \\
Renal toxicity G 3-4 & \\
Hematologic toxicity & \\
$\quad$ PMN G 3/4 & \\
$\quad$ Platelet G 3/4 & $7 / 1(13 / 1.8)$ \\
Liver toxicity (elevated transaminases, G 3/4) & $10 / 1(18 / 1.8)$
\end{tabular}

(3-5 $\mu \mathrm{g} / \mathrm{kg} / \mathrm{min}$ i.v.) for oliguria not responsive to fluid bolus, and for fluid overload manifested by pulmonary congestion or weight gain $>10 \%$. Ondansetron/granisetron or metoclopramide were used to alleviate symptoms of nausea and vomiting. Diphenoxylate or loperamide was used to alleviate diarrhea. Acetaminophen was given at a dose of $1000 \mathrm{mg}$ orally, every $6 \mathrm{~h}$ to control flu-like symptoms associated with biotherapy. Promethazine was used to control prolonged chills; in addition, antihistamines were given to prevent pruritus. No steroid treatment was allowed during the entire treatment period.

\section{Results}

Patients characteristics and dose intensity. The characteristics of 55 of the patients whose data was fully available and participated in this study are summarized in Table II. All patients had metastatic disease, and no patient had received any prior systemic therapy for metastatic disease.

A total of 205 courses of CBT were given, median of four courses, range of 2-8. Twenty percent of the patients were removed at some point from the treatment protocol due to toxicity or refusal to continue. Treatment delays of more than seven days occurred in $15 \%$ of the courses, usually in later cycles. The majority of the delays were due to unresolving thrombocytopenia; $17 \%$ of the treatment courses were stopped or modified due to toxicity.

Toxicity. Toxicity of the treatment regarding the 55 patients whose data was fully available is summarized in Table III. All patients experienced flu-like symptoms of varying degrees. Weight gain due to fluid retention during therapy 


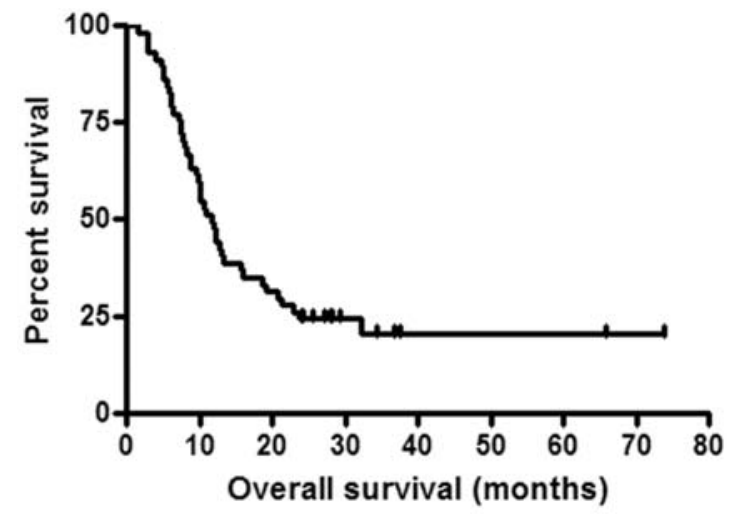

Figure 1. Survival of all patients who received at least two courses of biochemotherapy. Median survival was 11.7 months.

Table IV. Characteristics of $14 \mathrm{CR}$ patients.

Median (range)

Age

$47.5(31-71)$

Male/Female

$6 / 8$

PS

Previous adjuvant treatments

$\begin{array}{ll}\text { Radiotherapy } & 3 \\ \text { INF- } \alpha 2 b & 1 \\ \text { Vaccine } & 2\end{array}$

Number of metastatic sites

$\begin{array}{ll}1 & 5 \\ 2 & 5 \\ 3 & 3 \\ 4 & 1\end{array}$

Distribution of metastasis

Bone

Lung

Liver

Adrenal

Number of CBT courses given $5(3-8)$

was observed in all patients to various extents, mostly $<10 \%$ of body weight. Pulmonary congestion accompanied by hypoxemia was common, but no patients suffered respiratory failure requiring assisted ventilation. Although systolic blood pressure was below $90 \mathrm{mmHg}$ frequently, symptomatic hypotension was rare. Acute irreversible renal failure developed in one patient following the first course of therapy requiring dialysis, and one patient developed grade 3 transient renal failure. Mild reversible rise in creatinine (median $12.5 \%$ above baseline) was observed in $73 \%$ of patients.

Liver function abnormalities were common and reversible, and had no clinical consequences. Electrolyte imbalance was

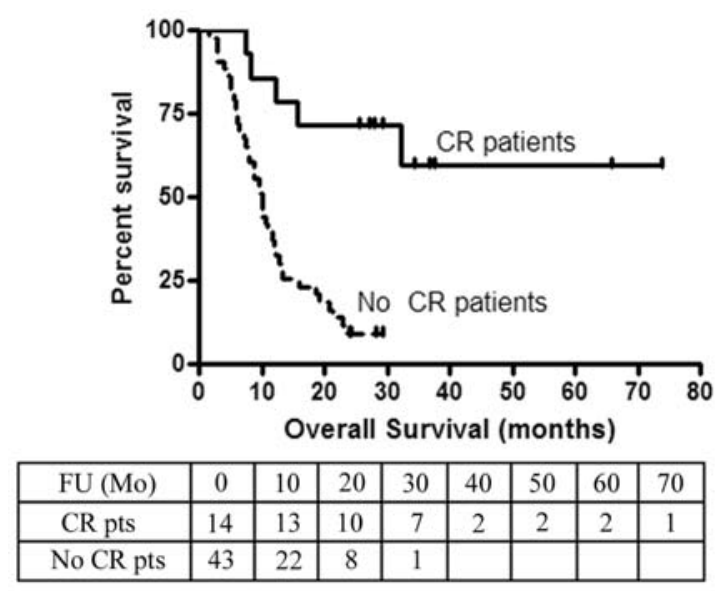

Figure 2. Survival of CR patients (median survival not reached), and nonCR patients (median survival 10 months), $\mathrm{P}<0.0001$ for comparison (logrank test), Hazard ratio 0.199 . The lower table presents the number of patients (pts) at risk.

treated with replacement therapy during hospitalization. Diarrhea was usually mild to moderate. This toxicity was managed symptomatically and was reversible in all patients.

Skin toxicity in the form of varying degrees of rash developed in the majority of patients. Dry skin and exfoliation required skin moisturizers. Pruritus was managed symptomatically.

Hematologic toxicity was usually moderate, but cumulative and frequent. There was only one episode of neutropenic fever, and two C. difficile infections were documented. Routine antibiotic treatment was not given. Four patients required blood transfusion, and two patients received platelet transfusion. There were no thrombocytopenia related bleeding events. No patient required intensive care unit admission, and there were no treatmentrelated deaths.

Outcome. Data referring to 57 patients in this study who completed at least two cycles of therapy are presented. The median follow-up of patients alive at the time of data analysis was 29 months (range 24-73 months). In two of our patients, CT findings at the end of CBT led to surgical treatment (adrenalectomy) that revealed no viable tumor cells. In one patient a residual soft tissue mass regressed completely following an additional course of s.c. INF. In one patient abnormal liver uptake was found in a PET scan, but this patient refused to repeat imaging studies, a palpable sub-cutaneous mass regressed completely, and the patient is with no clinical evidence of disease for more than two years. This patient was regarded in our analysis as having achieved CR.

The overall RR reached 44\% (25/57 patients); 14 patients had a CR $(25 \%) ; 11(19 \%)$ reached a PR. Median OS was 11.7 months for the 57 evaluable patients (Fig. 1). The median OS for the 14 patients who achieved CR has not been reached (range 7.5-74 months). No specific characteristics such as age, low burden of disease, or previous adjuvant treatments, were identified in the small group of CR patients (Table IV). OS for all other patients was 10 months. Log-rank comparison of OS of CR patients 


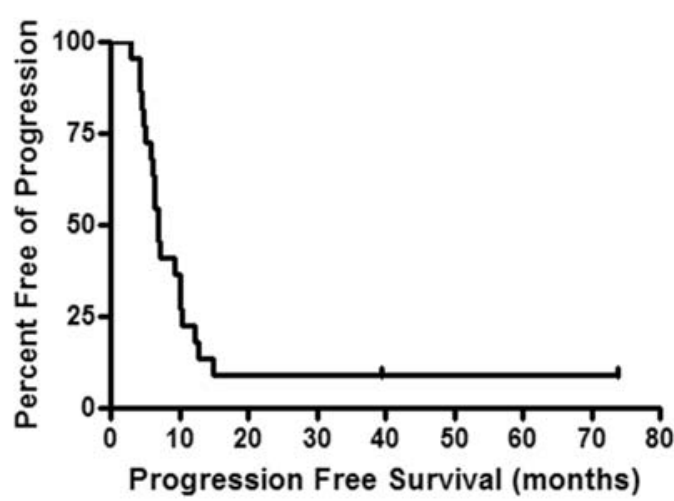

Figure 3. Progression-free survival of all patients who received at least two courses of biochemotherapy. Median PFS was 7 months.

compared to all other patients was statistically significant $(\mathrm{p}<0.0001$, Hazard ratio 0.199, Fig. 2). The PFS was 7 months for all evaluable patients (Fig. 3).

Eight of the $14 \mathrm{CR}$ patients (14\% of assessable patients, $57 \%$ of CR patients) are currently alive (range: $26-74$ months) with no clinical evidence of disease. Two patients that achieved CR developed later brain metastasis, and were rendered free of disease by radiotherapy in one case, or surgery, radiotherapy and chemotherapy in another case. In one of these patients brain metastasis recurred a second time 3 years after the treatment of the first brain relapse, and a NED status was achieved again by surgical resection. Aside from the second brain recurrence in the patient mentioned above, these 8 patients have remained diseasefree with no treatment for over two years.

\section{Discussion}

The most important endpoint of CBT given to patients suffering from metastatic melanoma is achieving a CR. All our long-term survivors were patients who experienced CR. More than half of the patients who achieved CR have remained free of disease for over two years. We report a rate of $\mathrm{CR}$ which is high relative to other reports. Predictive factors for CR are lacking. Prior chemotherapy exposure and brain metastasis have been reported by some to be a negative predictive factor for response $(4,8)$, but we had no such patients in our cohort. The clinical characteristics of our CR patients reveal a wide spectrum of ages, burden of disease, and sites of metastasis. Owing to the relatively small number of subjects, we were unable to identify predictors for CR in this study.

Interestingly, one of the long-term responders had an abnormal PET-CT finding which did not evolve to a clinical relapse during a follow-up of more than two years. This may represent fibrotic remnants with no viable tumor. Alternatively, the CBT treatment may have activated an anti-melanoma immune long-term response that keeps the tumor under control.

Our results compare favorably to those reported with high dose IL-2 (3). The clinical efficacy of the concurrent CBT protocol in our study was similar to the concurrent regimen of Legha et al with IL-2 (8). To the best of our knowledge, no head-to-head comparison between high dose IL-2 and CBT for metastatic melanoma has been performed. The existing data suggest that concurrent CBT achieves a higher overall RR compared to high-dose IL-2. However, the most important endpoint, the long-term remission rate, is similar for the two regimens (19).

An important drawback of most CBT treatments is the high rate of CNS failures. The CNS is a sanctuary site for this treatment. Protocols including temozolomide are being evaluated, with encouraging preliminary results $(20,21)$. Brain relapse is a harbinger of uncontrollable disease; we therefore consider only the CR patients that are alive and with no history of brain relapse as having a potential for long-term DFS, and possible cure.

The toxicity of our modified protocol is comparable to that of other reported concurrent CBT treatments $(4,16,22-24)$. We have used a decrescendo schedule of IL-2 administration, as suggested by Keilholz et al, which is associated with reduced toxicity compared to the administration of the same total amount of IL-2 in equally divided doses. This regimen was associated with enhanced RR (16). We did not observe any significant neurotoxicity, which is a commonly reported side effect of regimens that include vinca alkaloids $(8,9)$. We have avoided the use of central venous access in most cases. This may explain the low incidence of infectious complications, compared to the rate of $42 \%$ in other reports (4). The CBT protocol reported here, had a low toxicity profile with similar clinical efficacy as high-dose regimens, in terms of durable $\mathrm{CR}$ rate.

Granulocyte-macrophage stimulating factor (GM-CSF) enhances immune responses by inducing the proliferation, maturation, and migration of dendritic cells, and the expansion and differentiation of B and T lymphocytes. Due to its immune modulation effects and low toxicity profile, GM-CSF has become an attractive vaccine adjuvant, and a treatment modulator. Based on our previous experience (25), GM-CSF was added as an immunomodulator to the CBT of the first 26 patients. It was not used after that due to technical difficulties in acquisition of the drug. Comparing the two cohorts, those who received GM-CSF containing regimen and those who did not, did not reveal any differences in the clinical outcome. However, this study was not designed to test the efficacy of adding GM-CSF to CBT, and no conclusion can be derived regarding this issue.

Our results indicate that a certain sub-group of patients benefits significantly from the CBT combination. Importantly, $10 \%$ of our patients may be considered cured from metastatic melanoma. We believe the durability of the response seen in a fraction of the patients who achieved CR in our series, should not be disregarded. Median OS is the commonly used endpoint in clinical studies, but this method ignores the outliers. These are the values which are far away from the average, including the few long-term survivors. This may be considered an important endpoint in the study of a disease with a negligible cure rate such as metastatic melanoma.

In view of our results, which are similar to those reported for high-dose bolus IL-2, we suggest to design a phase III study comparing high dose IL-2 to CBT. In the meantime, we suggest the use of our CBT protocol as a feasible first line, aggressive palliative treatment, in patients with good 
performance status and no co-morbidities. Importantly, this treatment offers these patients a potential for cure. In the future, genetic profiling of tumors and patients may allow prediction of patients' response. This would alleviate the unnecessary toxicity from those who have only a slight chance of responding, and promote maximal dose intensity in the patients with a chance of complete response and possible cure.

\section{References}

1. Tsao H, Atkins MB and Sober AJ: Management of cutaneous melanoma. N Engl J Med 351: 998-1012, 2004.

2. Rosenberg SA, Yang JC, Topalian SL, et al: Treatment of 283 consecutive patients with metastatic melanoma or renal cell cancer using high-dose bolus interleukin 2. JAMA 271: 907913, 1994.

3. Atkins MB, Lotze MT, Dutcher JP, et al: High-dose recombinant interleukin 2 therapy for patients with metastatic melanoma: analysis of 270 patients treated between 1985 and 1993. J Clin Oncol 17: 2105-2116, 1999.

4. O'Day SJ, Gammon G, Boasberg PD, et al: Advantages of concurrent biochemotherapy modified by decrescendo interleukin-2, granulocyte colony-stimulating factor, and tamoxifen for patients with metastatic melanoma. J Clin Oncol 17: 2752-2761, 1999.

5. Casares N, Pequignot MO, Tesniere A, et al: Caspasedependent immunogenicity of doxorubicin-induced tumor cell death. J Exp Med 202: 1691-1701, 2005.

6. Lake RA and Robinson BW: Immunotherapy and chemotherapy-a practical partnership. Nat Rev Cancer 5: 397-405, 2005.

7. Lake RA and van der Most RG: A better way for a cancer cell to die. N Engl J Med 354: 2503-2504, 2006.

8. Legha SS, Ring S, Eton O, et al: Development of a biochemotherapy regimen with concurrent administration of cisplatin, vinblastine, dacarbazine, interferon alfa, and interleukin-2 for patients with metastatic melanoma. J Clin Oncol 16:1752-1759, 1998.

9. Eton O, Legha SS, Bedikian AY, et al: Sequential biochemotherapy versus chemotherapy for metastatic melanoma: Results from a phase III randomized trial. J Clin Oncol 20: 2045-2052, 2002.

10. Keilholz U, Goey SH, Punt CJ, et al: Interferon alfa-2a and interleukin-2 with or without cisplatin in metastatic melanoma: a randomized trial of the European Organization for Research and Treatment of Cancer Melanoma Cooperative Group. J Clin Oncol 15: 2579-2588, 1997.

11. Rosenberg SA, Yang JC, Schwartzentruber DJ, et al: Prospective randomized trial of the treatment of patients with metastatic melanoma using chemotherapy with cisplatin, dacarbazine, and tamoxifen alone or in combination with interleukin-2 and interferon alfa-2b. J Clin Oncol 17: 968-975, 1999.

12. Ridolfi R, Chiarion-Sileni V, Guida M, et al: Cisplatin, dacarbazine with or without subcutaneous interleukin-2, and interferon alpha- $2 b$ in advanced melanoma outpatients: results from an Italian multicenter phase III randomized clinical trial. J Clin Oncol 20: 1600-1607, 2002.
13. Bajetta E, Del Vecchio M, Nova P, et al: Multicenter phase III randomized trial of polychemotherapy (CVD regimen) versus the same chemotherapy (CT) plus subcutaneous interleukin-2 and interferon-alpha2b in metastatic melanoma. Ann Oncol 17: 571-577, 2006

14. Ives NJ, Stowe RL, Lorigan P and Wheatley K: Chemotherapy compared with biochemotherapy for the treatment of metastatic melanoma: a meta-analysis of 18 trials involving 2,621 patients. J Clin Oncol 25: 5426-5434, 2007.

15. Flaherty LE, Atkins M, Sosman J, et al: Outpatient biochemotherapy with interleukin-2 and interferon alfa- $2 b$ in patients with metastatic malignant melanoma: results of two phase II cytokine working group trials. J Clin Oncol 19: 3194-3202, 2001

16. Keilholz U, Scheibenbogen C, Tilgen W, et al: Interferon-alpha and interleukin-2 in the treatment of metastatic melanoma. Comparison of two phase II trials. Cancer 72: 607-614, 1993.

17. O'Day SJ, Boasberg PD, Piro L, et al: Maintenance biotherapy for metastatic melanoma with interleukin-2 and granulocyte macrophage-colony stimulating factor improves survival for patients responding to induction concurrent biochemotherapy. Clin Cancer Res 8: 2775-2781, 2002.

18. Chang DZ, Lomazow W, Joy Somberg C, Stan R and Perales MA: Granulocyte-macrophage colony stimulating factor: an adjuvant for cancer vaccines. Hematology 9: 207-215, 2004

19. Keilholz U and Gore ME: Biochemotherapy for advanced melanoma. Semin Oncol 29: 456-461, 2002.

20. Weber RW, O'Day S, Rose M, et al: Low-dose outpatient chemobiotherapy with temozolomide, granulocyte-macrophage colony stimulating factor, interferon-alpha $2 \mathrm{~b}$, and recombinant interleukin-2 for the treatment of metastatic melanoma. J Clin Oncol 23: 8992-9000, 2005.

21. Ron IG, Sarid D, Ryvo L, et al: A biochemotherapy regimen with concurrent administration of cisplatin, vinblastine, temozolomide (Temodal), interferon-alfa and interleukin-2 for metastatic melanoma: a phase II study. Melanoma Res 16: 65-69, 2006.

22. Legha SS, Ring S, Eton O, Bedikian A, Plager C and Papadopoulos N: Development and results of biochemotherapy in metastatic melanoma: the University of Texas M.D. Anderson Cancer Center experience. Cancer J Sci Am 3 (Suppl 1): S9-S15, 1997.

23. Thompson JA, Gold PJ and Fefer A: Outpatient chemoimmunotherapy for the treatment of metastatic melanoma. Semin Oncol 24: S44-S48, 1997.

24. Thompson JA, Gold PJ, Markowitz DR, Byrd DR, Lindgren CG and Fefer A: Updated analysis of an outpatient chemoimmunotherapy regimen for treating metastatic melanoma. Cancer J Sci Am 3 (Suppl 1): S29-S34, 1997.

25. Schachter J, Rakowsky E, Sulkes A and Adler A: A sequential four-drug chemotherapy and biotherapy with interferon alpha and GM-CSF-an innovative protocol for the treatment of metastatic melanoma. Cancer Biother Radiopharm 13: 155-164, 1998. 